LEADING ARTICLE

\title{
Proton pump inhibitors and gastric neoplasia
}

\author{
E J Kuipers
}

Gut 2006;55:1217-1221. doi: 10.1136/gut.2005.090514

Proton pump inhibitors (PPIs) are very effective in maintaining symptomatic and endoscopic remission of acid peptic disorders, such as gastro-oesophageal reflux disease. Side effects with respect to function and morphology of the gastric mucosa are common. Helicobacter pylori eradication can partially prevent and reverse these effects without impairing PPI therapy for gastro-oesophageal reflux disease. This makes long term PPI treatment a safe therapy for patients with acid peptic disorders. The potential side effects of such therapy are discussed here.

Correspondence to:

Dr E J Kuipers, Department of Gastroenterology and Hepatology, Erasmus MC University Medical Centre, PO Box 2040, 3000 CA Rotterdam, the

Netherlands; e.j.kuipers@ erasmusmc.nl

Received 4 May 2006 Accepted for publication 9 May 2006
$\mathrm{P}$ roton pump inhibitors (PPIs) have become a major asset in our pharmacological armamentarium over the past 15 years. These drugs are very efficacious for the treatment of acid peptic disorders, conditions which are common throughout the world. In Asia and developing countries, peptic ulcer disease is still the most common acid peptic disorder, although the incidence of this condition is changing. In the West, gastro-oesophageal reflux disease (GORD) is now far more common than peptic ulcer disease. In a Canadian primary study, 43\% of patients presenting with dyspepsia were diagnosed with oesophagitis and 5\% with peptic ulcer disease. ${ }^{1}$ In a recent survey in a population of 500000 primary care patients, we observed that the annual incidence of new cases of Barrett's oesophagus had more than doubled in the period from 1996 to 2003, ${ }^{2}$ also demonstrating the very common presence and increasing prevalence of GORD and its associated complications. In all of these conditions, PPIs are the most effective acid suppressive treatment. In addition, widespread use of non-steroidal anti-inflammatory drugs in populations at risk of gastroduodenal side effects is another common indication for PPI therapy. In the Netherlands, $21 \%$ of PPI prescriptions are given for gastroprotection to non-steroidal anti-inflammatory drug or aspirin users. ${ }^{3}$ The use of PPIs is thus increasing and this increase may continue over the coming years for several reasons. Reasons include the further rise in the prevalence of GORD and the availability of over the counter and generic PPIs. Furthermore, studies have shown that $70-80 \%$ of non-steroidal anti-inflammatory drug users at risk for gastroduodenal complications do not yet receive gastroprotection. $^{45}$ This may explain the unchanging incidence of complicated ulcer disease despite a decrease in the prevalence of Helicobacter pylori. $^{6}$
In the Dutch population, the percentage of subjects who use at least one PPI prescription per year is now approximately $4 \%$. The majority of these patients use a PPI for a short period, more than $50 \%$ stop within the first year of treatment, but approximately one third continue for more than two years. ${ }^{3}$ Hence our experience of maintenance therapy with PPIs is now 22 years of continuous treatment, in particular in patients with severe reflux disease. Given the high incidence of acid related disorders and the excellent efficacy of PPIs for the treatment of these conditions, the safety of long term PPI treatment is very relevant.

This review focuses on PPI therapy and the development and diagnosis of gastric neoplasia. PPIs and the development of gastric neoplasia relate to hypergastrinaemia and gastritis. PPIs and the diagnosis of gastric neoplasia relates to symptoms and the accuracy of diagnostic endoscopy.

\section{PPIs, HYPERGASTRINAEMIA, AND \\ GASTRIC NEOPLASIA}

\section{PPIs and gastric carcinoids}

Profound acid suppressive therapy leads to hypergastrinaemia in nearly all patients. In the majority, serum gastrin levels are only moderately increased $(<400 \mathrm{ng} / \mathrm{l}$ or $<4$ times the upper limit of normal). In most of these patients, serum gastrin levels quickly normalise after withdrawal of PPI therapy. In some patients however gastrin levels during PPI therapy can increase to higher levels (400-4000 ng/l) and may show slower or incomplete normalisation after PPI withdrawal. ${ }^{7}$ The latter group are more often infected with $H$ pylori and have a high prevalence of atrophic gastritis of the gastric body mucosa.

\section{"Profound acid suppressive therapy leads to hypergastrinaemia in nearly all patients"}

When PPIs were introduced, the sustained hypergastrinaemia due to PPI maintenance therapy raised concern. In rats, prolonged hypergastrinaemia as a result of profound acid suppression results in hyperplasia of enterochromaffin-like cells. This can ultimately lead to gastric carcinoid formation. ${ }^{8}$ However, this phenomenon has never been observed in other species. In humans, diffuse, linear, or micronodular hyperplasia of enterochromaffin-like

Abbreviations: PPI, proton pump inhibitor; GORD, gastro-oesophageal reflux disease; FGP, fundic gland polyps; FAP, familial adenomatous polyposis; IPCl database, Dutch Integrated Primary Care Information database 
cells is observed in 10-30\% of chronic PPI users, in particular in $H$ pylori positive patients with more markedly increased gastrin levels. ${ }^{9}$ Most of these patients have moderate to severe inflammation of the body mucosa, often with atrophic changes. The apparent enterochromaffin-like cell hyperplasia may indicate an actual increase in these cells but it may also be the result of their clustering in an otherwise atrophic mucosa ("pseudo hyperplasia"). Most importantly, dysplasia or invasive carcinoid formation has never been described in long term PPI users and thus is not an indication for surveillance in PPI maintenance users.

\section{PPIs and fundic gland polyps}

PPI induced hypergastrinaemia has also been associated with the development of fundic gland polyps (FGPs). These lesions are the most common polyps in the stomach and are usually sessile, rather weak on contact, and only located in the acid secreting corpus mucosa. Histologically, they consist of distorted glandular cysts lined with fundic-type epithelial cells. FGPs are common in patients with familial adenomatous polyposis (FAP), and also occur in other patients as sporadic FGPs. A relation between sporadic FGPs and PPI therapy has been questioned but most of the literature suggests a rather strong association between these polyps and PPI maintenance therapy. ${ }^{10}$ The exact aetiology of sporadic FGPs is unknown although an association with $\beta$-catenin gene mutations has been described. ${ }^{11}{ }^{12}$ The majority of PPI associated FGPs are however clearly associated with hypergastrinaemia leading to parietal cell hyperplasia with protrusion into the lumen of the gland. This leads to narrowing of the glandular lumen and cystic dilation of the gland. ${ }^{10}$ We therefore proposed the hypothesis that these polyps are mainly the mechanistic result of impaired glandular flow. ${ }^{10}$ This is supported by the fact that dysplastic changes in FGPs developing during PPI therapy are very rare and have only been reported in a few individual cases, despite the frequency of these polyps during PPI therapy. ${ }^{13}{ }^{14}$ Dysplasia is common in FAP associated FGPs, with a prevalence ranging from $2 \%$ to $53 \%$ in various series. ${ }^{13}$ FGPs with and without dysplasia have been described in FAP patients on PPI therapy, ${ }^{15}$ but it is not known whether PPI therapy increases the risk of FGPs and dysplasia in FAP patients. Apart from FAP patients, a diagnosis of FGP during PPI therapy does not warrant endoscopic removal of polyps, nor does it require surveillance or withdrawal of PPI therapy, given the fact that these lesions do not progress and only seem to develop dysplastic changes in extremely rare cases.

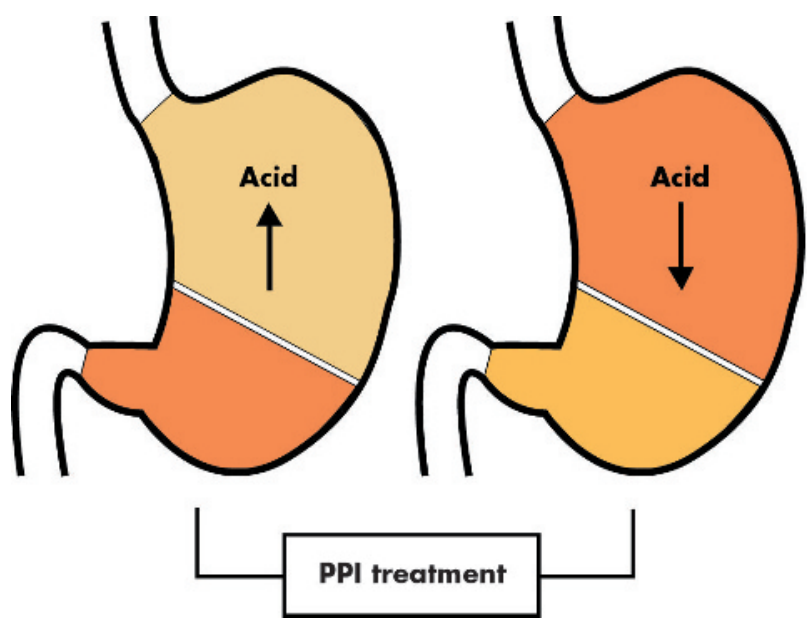

Figure 1 Proton pump inhibitor (PPI) therapy in Helicobacter pylori positive patients changes the gastritis pattern from antral predominant to corpus predominant pan-gastritis.
PPIs, GASTRITIS, AND GASTRIC NEOPLASIA

Acid secretion and Helicobacter pylori colonisation Discussion on the long term safety of PPIs initially focused on gastrin but the main interest has in the past decade shifted to PPIs and gastritis. The key issue in the understanding of this topic is the interrelation between acid secretion and $H$ pylori colonisation. In subjects in whom acid production is intact, $H$ pylori predominantly colonises the gastric antrum. This colonisation pattern is associated with an antral predominant gastritis. Inflammation of the antral mucosa stimulates gastrin secretion which maintains acid production at a normal to high level and thus keeps the pattern intact. In contrast, in subjects in whom acid production is decreased by whatever mechanism, including the use of PPIs, $H$ pylori also colonises the body of the stomach, leading to a corpus predominant gastritis (fig 1). Inflammation of the gastric corpus mucosa further impairs acid secretion despite the increase in gastrin as a result of both the concomitant inflammation of the antrum and the reduction in acid secretion. Again the circle is kept intact. The occurrence of body gastritis with impairment of parietal cell function augments the acid suppressive effect of the PPI. ${ }^{16}$ This effect, although measurable in intragastric $\mathrm{pH}$ studies, is of limited magnitude in comparison with the overall effect of the PPI itself, and therefore has only limited clinical consequences. It appears only in the first few weeks of treatment when PPIs have a higher efficacy for healing of erosive oesophagitis in $H$ pylori positive than in $H$ pylori negative patients.

\section{"H pylori status is not an issue in the dosing of a PPI for an individual patient"}

In a large study from Germany, investigators found an approximate $10 \%$ difference $(86 \% \vee 76 \%$ ) in the healing rates for $H$ pylori negative and positive patients with erosive oesophagitis when treated with pantoprazole $40 \mathrm{mg}$ once daily for four weeks. ${ }^{17}$ This difference however disappeared after eight weeks of treatment. Likewise, a long term maintenance study failed to find a difference in the efficacy of PPI maintenance treatment between $H$ pylori positive and negative patients. ${ }^{9}$ From these studies, one can conclude that the initial acid suppressive effect of PPIs is somewhat higher in $H$ pylori positive than in $H$ pylori negative patients as a result of the augmenting effect of corpus gastritis further impairing acid secretion. Yet, the clinical relevance of this phenomenon is very limited and $H$ pylori status is thus not an issue in the dosing of a PPI for an individual patient.

\section{Long term consequences of corpus predominant gastritis}

The pattern of corpus predominant gastritis persists throughout treatment with a PPI in $H$ pylori positive patients. The possible consequences of this phenomenon have been the issue of much debate over the past decade. Firstly, it was consistently found that the incidence of atrophic gastritis was higher in subjects with a condition effecting acid secretion, such as gastric ulcer disease, previous vagotomy, or PPI maintenance treatment, than in the general population. ${ }^{18-24}$ We therefore studied the development of atrophic gastritis in two populations of GORD patients: one treated with fundoplication without further acid suppressive therapy and one treated with omeprazole maintenance therapy. We found that the incidence of atrophic gastritis was very low in $H$ pylori negative cases irrespective of the modality of treatment, and also in $H$ pylori positive patients when treated with fundoplication. ${ }^{25}$ However, in $H$ pylori positive cases treated with omeprazole maintenance therapy, a steady gradual development of atrophic gastritis was observed within the first years of treatment so that after an average of five years 
of treatment approximately one out of every three patients had developed this condition. ${ }^{25}$ In subsequent years, these findings were claimed to be refuted by another study with a randomised but otherwise similar design following GORD patients who were treated with either fundoplication or omeprazole maintenance therapy. ${ }^{26}$ However, when carefully comparing both studies, it seems that both showed very similar patterns of changes, both with respect to induction of a corpus predominant gastritis and the rate of development of atrophic changes in $H$ pylori positive patients treated with omeprazole. The fact that the second study was nevertheless unable to confirm a significant difference in the progression towards atrophic gastritis between $H$ pylori positive patients treated with fundoplication versus those treated with omeprazole could be fully attributed to the lack of power of this study which included fewer patients and had a shorter follow up time. ${ }^{27-29}$

\section{"The pattern of corpus predominant gastritis persists throughout treatment with a PPI in H pylori positive patients"}

In a recent update of the same study, now with seven years follow up, the authors confirmed a similar progression towards atrophic gastritis in $H$ pylori positive patients treated with acid suppression as previously noted by others. ${ }^{30}$ The immediate effect of PPI therapy on the distribution of $H$ pylori gastritis invariably has been confirmed in all studies on this topic. The progression towards atrophic gastritis has also been confirmed in most other PPI studies, ${ }^{93} 32$ but not in all. ${ }^{33}{ }^{34}$ The main difference between these studies was the duration of follow up, being considerable longer in the studies which observed progression towards atrophy in $H$ pylori positive patients.

The debate on the long term consequences of PPI treatment in $H$ pylori positive patients also focussed on the actual histological aspects of atrophic gastritis. Further studies confirmed that this was a true loss of glands instead of a false interpretation of inflammation. Firstly, this was based on different kinetics of inflammation and atrophy, with body inflammation occurring immediately after the start of PPI therapy and atrophy only developing over several years. Secondly, other studies showed that atrophy but not corpus gastritis by itself lowered serum vitamin B12 levels and was associated with very high gastrin levels during omeprazole maintenance therapy. ${ }^{75}$ In one follow up study of GORD patients using PPI maintenance treatment, we found that some developed very high gastrin levels (440 times the upper limit of normal). ${ }^{7}$ These patients were nearly all infected with $H$ pylori and approximately 55\% had signs of atrophic gastritis. In general, monitoring of serum gastrin levels and serum vitamin B12 levels is not considered useful in PPI maintenance users.

\section{Other factors potentially affecting gastric cancer risk} Several studies have also addressed the fact that profound acid suppressive therapy can be associated with bacterial overgrowth with non-Helicobacter species within the stomach. ${ }^{36}$ It seems that this is more common in $H$ pylori positive patients, in particular in the presence of atrophic gastritis. This bacterial overgrowth has been associated with more severe gastritis and increased serum cytokine levels. ${ }^{36}$ The clinical importance of these observations remains to be evaluated. In particular, it is not known whether bacterial overgrowth is a consequence of atrophic gastritis in PPI users or a contributing aetiological factor.

\section{Gastric cancer in PPI users}

The development of atrophic gastritis in $H$ pylori positive subjects with low acid production has been a consistent observation. The long term consequences, in particular with respect to the risk of gastric cancer development, require further study. The available cohort studies in chronic PPI users do not provide data with gastric cancer as the end point. This can be explained simply by the lack of sufficient power of those studies with respect to both the number of subjects included and the period of follow up. In addition, even if the cohorts were larger, they would still lack adequate controls with the same acid peptic condition and followed in the same manner but not treated with a PPI. In the absence of such controlled cohort data, one may look for observational data from large population databases.

\section{"The development of atrophic gastritis in $H$ pylori positive subjects with low acid production has been a consistent observation"}

One such database is the Dutch Integrated Primary Care Information (IPCI) database. This general practice research database contains the computer based medical records of more than 500000 patients in the Netherlands. This dynamic database was started in 1992 and has since expanded. The IPCI population has the same sex and age distribution as the Dutch general population The database contains the records of 27328 patients who used at least one PPI prescription. During eight years of follow up, $45(0.16 \%)$ were diagnosed with gastric cancer compared with $22(0.01 \%)$ gastric cancer cases among 358000 subjects not using a PPI and followed for at least one year (Eva van Soest, unpublished data). This may at first glance seem a striking difference but from these data one can only conclude that PPI treatment does not protect against gastric cancer, a finding that is not surprising. The conclusion that PPI treatment increases the risk for gastric cancer development is not justified, as there are at least two important confounders that can fully explain the observation of a higher gastric cancer incidence among PPI users than controls. The first is protopathic bias (that is, PPIs were prescribed for initial symptoms related to the already present cancerous lesion). This is supported by the observation that the ratio of observed versus expected gastric cancer cases was the highest in the first 12 months after the start of PPI therapy. The second important bias may come from confounding by indication (that is, PPIs are given for conditions which by themselves increase the risk of gastric cancer, such as dyspeptic complaints related to $H$ pylori infection and gastric ulcer disease).

\section{"PPI therapy affects the pattern and severity of $H$ pylori} gastritis and accelerates the process of corpus gland loss"

In summary, PPI therapy affects the pattern and severity of $H$ pylori gastritis and accelerates the process of corpus gland loss. At present there is no proof that this increases the risk of gastric cancer. There are however circumstantial data both from $\operatorname{Japan}^{37}$ and Europe ${ }^{38}$ that persistent corpus predominant gastritis and atrophy are major risk factors for the development of gastric cancer. Together, the Maastricht consensus panel on the management of $H$ pylori infection therefore concluded that acid suppression affects the pattern and distribution of gastritis, favouring corpus predominant gastritis. ${ }^{39}$ This may accelerate the process of loss of specialised glands leading to atrophic gastritis, and therefore the advice of the consensus panel was to consider $H$ pylori eradication in infected subjects requiring long term maintenance treatment with a PPI. ${ }^{39}$

\section{Effect of $\boldsymbol{H}$ pylori eradication}

The question now becomes, what is the effect of $H$ pylori eradication in long term PPI users? The first study to answer 
this was performed in a small population. It showed that development of atrophic gastritis could be prevented by $H$ pylori eradication at the start of one year of omeprazole therapy for GORD. ${ }^{40}$ In a further two year cohort study in patients who, on average, had already received a five year treatment course of omeprazole for GORD, we observed that eradication of $H$ pylori led to resolution of corpus gastritis and regression of atrophic gastritis, even when this condition was already present in a more severe form ${ }^{41}$ Healing of gastritis and resolution of atrophy was not associated with the need for a higher dose of PPI. We thus concluded that $H$ pylori eradication induces regression of gastric mucosal inflammation and atrophy in chronic PPI uses, without affecting the efficacy of PPI therapy for GORD. ${ }^{41}$

\section{"H pylori eradication induces regression of gastric mucosal inflammation and atrophy in chronic PPI uses, without affecting the efficacy of PPI therapy for GORD"}

\section{PROFOUND ACID SUPPRESSION AND THE DIAGNOSIS OF GASTRIC CANCER}

Empirical PPI therapy is widely used and advocated in various guidelines. However, concern is that empirical treatment may interfere with the diagnosis of gastric cancer leading to diagnostic delay, impairing treatment options and survival. This may occur in two ways. Firstly, the use of acid suppressors may reduce symptoms and thus delay diagnostic endoscopy. Secondly, when endoscopy is performed, treatment with a PPI may conceal visualisation of early gastrooesophageal cancer and lead to a false negative endoscopy. With respect to the first issue, most guidelines advise limiting the duration of empirical treatment, in particular in elderly subjects, and to refer patients with alarm symptoms for immediate endoscopy without prior empirical therapy. Unfortunately, there are discrepancies between guidelines and clinical practice. An interesting primary care study from the UK showed that $20 \%$ of patients with alarm symptoms in the presence of an undiagnosed gastric or oesophageal adenocarcinoma received empirical acid suppressive treatment instead of an immediate referral for endoscopy. ${ }^{42}$ As expected, this treatment did not have an effect on those symptoms and later referral occurred. Furthermore, the correlation between gastro-oesophageal cancer and alarm symptoms is limited. In particular, many patients with gastric cancer present with non-specific complaints. These patients are likely to receive empirical PPI treatment. In the study from the UK, 52\% of 339 patients with gastro-oesophageal cancer and benign symptoms had received antisecretory treatment between the onset of symptoms and diagnosis. ${ }^{42}$ In other European studies, $43-53 \%$ of large cohorts of patients with gastro-oesophageal cancer had received an antisecretory drug prior to the first endoscopy..$^{43}{ }^{44}$ Obviously, this may delay the timing of diagnostic endoscopy. In one study which specifically addressed this, antisecretory treatment led to a mean 3.9 months delay before endoscopy was performed. ${ }^{42}$

\section{"Concern is that empirical treatment may interfere with the diagnosis of gastric cancer leading to diagnostic delay, impairing treatment options and survival"}

A second issue is whether PPI treatment increases the risk of obscuring the diagnosis of cancer when endoscopy is performed. PPI treatment may lead to re-epithelialisation of a malignant ulcer. ${ }^{43}{ }^{45}$ A small case series described complete resolution of endoscopic abnormalities in seven patients with ulcerated early gastric cancer who received a course of PPIs while awaiting a second confirmatory endoscopy. ${ }^{45}$ In one cohort study of 116 patients with gastro-oesophageal adenocarcinoma, prior acid suppressive treatment was clearly associated with failure to reach a diagnosis at first endoscopy. ${ }^{43}$ The diagnosis of cancer or a precancerous lesion had been missed at first endoscopy in only one $(2 \%)$ of 54 patients not receiving acid suppressive treatment, two (12\%) of 17 patients receiving a $\mathrm{H}_{2}$ blocker, and 20 (44\%) of 45 patients receiving PPI treatment. ${ }^{43}$ Fifteen of those 20 patients were diagnosed with gastro-oesophageal cancer within two years after the index endoscopy. These alarming findings were contradicted in a recent large cohort study from Denmark focusing on 27829 patients undergoing a first diagnostic gastroscopy. ${ }^{44}$ Gastro-oesophageal cancer was diagnosed at this index endoscopy in 461 patients, and in another 52 patients during a median follow up of 2.7 years after the first endoscopy. Therefore, signs of cancer were missed at the index endoscopy in $11 \%$ of cancer patients. This risk was the same in users and non-users of a PPI. ${ }^{44}$ The authors concluded that very few cancers were missed during upper gastrointestinal endoscopy but the miss rate actually corresponded with a miss rate of one cancer per 536 endoscopies, or presumably one lesion per average endoscopist per year.

\section{"PPI therapy may delay diagnostic endoscopy and can in some settings also increase the risk of failure to diagnose cancer during endoscopy"}

In summary, one can conclude that PPI therapy may delay diagnostic endoscopy and can in some settings also increase the risk of failure to diagnose cancer during endoscopy. Nevertheless, cancer patients who previously received PPI treatment are diagnosed at a similar stage and have a similar prognosis as cancer patients who did not receive acid suppressive treatment. ${ }^{42}{ }^{44}$ This justifies the policy of empirical PPI therapy for patients with a first presentation of dyspepsia, if the duration of such therapy is limited and if patients with alarm symptoms are referred for immediate endoscopy.

\section{CONCLUSIONS}

PPIs are very effective in maintaining symptomatic and endoscopic remission of acid peptic disorders such as peptic ulcer and gastro-oesophageal reflux disease. Effects on function and morphology of the gastric mucosa are common by induction of both hypergastrinaemia and, in $H$ pylori positive patients, corpus predominant pan-gastritis. PPI therapy affects the pattern and severity of $H$ pylori gastritis and accelerates the process of corpus gland loss. At present there is no proof that this increases the risk of gastric cancer. However, persistent corpus predominant gastritis and atrophy are considered major risk factors for the development of gastric cancer. $H$ pylori eradication can partially prevent and reverse these effects without impairing PPI therapy for GORD. This makes long term PPI treatment safe for patients with acid peptic disorders but a $H$ pylori test and treat strategy needs to be considered in patients who require long term PPI therapy.

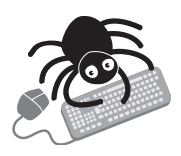

Conflict of interest: declared (the declaration can be viewed on the Gut website at http://www.gutjinl.com/ supplemental).

\section{REFERENCES}

1 Thomson AB, Barkun AN, Armstrong D, et al. The prevalence of clinically significant endoscopic findings in primary care patients with uninvestigated dyspepsia: the Canadian Adult Dyspepsia Empiric Treatment-Prompt Endoscopy (CADET-PE) study. Aliment Pharmacol Ther 2003;17:1481-91. 
2 van Soest EM, Dieleman JP, Siersema PD et al Increasing incidence of Barrett's oesophagus in the general population. Gut 2005;54:1062-6.

3 van Soest EM, Siersema PD, Dieleman JP, et al. Persistence and adherence to proton pump inhibitors in daily clinical practice. Aliment Pharmacol Ther 2006 (in press).

4 Abraham NS, El-Serag HB, Johnson ML, et al. National adherence to evidence-based guidelines for the prescription of nonsteroidal antiinflammatory drugs. Gastroenterology 2005; 129:1171-8.

5 Sturkenboom MC, Burke TA, Dieleman JP, et al. Underutilization of preventive strategies in patients receiving NSAIDs. Rheumatology (Oxford) 2003;42(suppl 3):iii23-31.

6 Post PN, Kuipers EJ, Meijer GA. Declining incidence of peptic ulcer, but not of its complications: a nation-wide study in The Netherlands. Aliment Pharmacol Ther 2006;23:1587-93.

7 Schenk BE, Kuipers EJ, Klinkenberg-Knol EC, et al. Hypergastrinaemia during long-term omeprazole therapy: influences of vagal nerve function, gastric emptying and Helicobacter pylori infection. Aliment Pharmacol Ther 1998;12:605-12.

8 Larsson $\mathrm{H}$, Carlsson E, Hakanson R, et al. Time-course of development and reversal of gastric endocrine cell hyperplasia after inhibition of acid secretion. Studies with omeprazole and ranitidine in intact and antrectomized rats. Gastroenterology 1988;95:1477-86

9 Klinkenberg-Knol EC, Nelis F, Dent J, et al. Long-term omeprazole treatment in resistant gastroesophageal reflux disease: efficacy, safety and influence on gastric mucosa. Gastroenterology 2000;1 18:661-9.

10 Cats A, Schenk BE, Bloemena E, et al. Parietal cell protrusions and fundic gland cysts during omeprazole maintenance treatment. Hum Pathol 2000;31:684-90.

11 Jalving M, Koornstra JJ, Boersma-van Ek W, et al. Dysplasia in fundic gland polyps is associated with nuclear beta-catenin expression and relatively high cell turnover rates. Scand J Gastroenterol 2003;38:916-22.

12 Watanabe N, Seno H, Nakajima T, et al. Regression of fundic gland polyps following acquisition of Helicobacter pylori. Gut 2002;51:742-5.

13 Jalving M, Koornstra JJ, Gotz JM, et al. High-grade dysplasia in sporadic fundic gland polyps: a case report and review of the literature. Eur J Gastroenterol Hepatol 2003;15:1229-33.

14 Wu T, Kornacki S, Rashid A, et al. Dysplasia and dysregulation of proliferation in foveolar and surface epithelia of fundic gland polyps from patients with familial adenomatous polyposis. Am J Surg Pathol 1998;22:293-8.

15 Attard TM, Yardley JH, Cuffari C. Gastric polyps in pediatrics: an 18-year hospital-based analysis. Am J Gastroenterol 2002;97:298-301.

16 Verdú $E$, Armstrong D, Fraser $R$, et al. Effect of $H$. pylori status on intragastric $\mathrm{pH}$ during treatment with omeprazole. Gut 1995;36:539-43.

17 Holtmann G, Cain C, Malfertheiner P. Gastric Helicobacter pylori infection accelerates healing of reflux esophagitis during treatment with the proton pump inhibitor pantoprazole. Gastroenterology 1999;117:11-16.

18 Kuipers EJ, Lee A, Klinkenberg-Knol EC, et al. Review article: The development of atrophic gastritis-Helicobacter pylori and the effects of acid suppressive therapy. Aliment Pharmacol Ther 1995;9:331-40.

19 Klinkenberg-Knol EC, Festen HPM, Jansen JBMJ, et al. Efficacy and safety of long-term treatment with omeprazole for refractory reflux esophagitis. Ann Intern Med 1994;121:161-7.

20 Solcia E, Fiocca R, Havu N, et al. Gastric endocrine cells and gastritis in patients receiving long-term omeprazole treatment. Digestion 1992:51:82-92.

21 Lamberts R, Creutzfeldt W, Strüber HG, et al. Long-term omeprazole therapy in peptic ulcer disease: gastrin, endocrine cell growth, and gastritis. Gastroenterology 1993;104:1356-70.

22 Havu N, Maaroos HI, Sipponen P. Argyrophil cell hyperplasia associated with chronic corpus gastritis in gastric ulcer disease. Scand J Gastroenterol 1991;26:90-4.

23 Peetsalu A, Maaroos $\mathrm{HI}$, Sipponen $\mathrm{P}$, et al. Long-term effect of vagotomy on gastric mucosa and Helicobacter pylori in duodenal ulcer patients. Scand J Gastroenterol 1991;26:77-83.
24 Brunner GHG, Lamberts R, Creutzfeldt W. Efficacy and safety of omeprazole in long-term treatment of peptic ulcer and reflux oesophagitis resistant to ranitidine. Digestion 1990;47:64-8.

25 Kuipers EJ, Lundell L, Klinkenberg-Knol EC, et al. Atrophic gastritis and Helicobacter pylori infection in patients with reflux esophagitis treated with omeprazole or fundoplication. N Engl J Med 1996;334:1018-22.

26 Lundell L, Havu N, Miettinen P, et al. No effect of acid suppression therapy over five years on gastric glandular atrophy. Results of a randomised clinical study. Gastroenterology 1999;118:A214.

27 McColl KEL, Murray LS, Gillen D. Omeprazole and accelerated onset of atrophic gastritis. Gastroenterology 2000;1 18:239.

28 Pounder RE, Williams MP. Omeprazole and accelerated onset of atrophic gastritis. Gastroenterology 2000;118:238-9.

29 Kuipers EJ, Klinkenberg-Knol EC, Meuwissen SGM. Omeprazole and accelerated onset of atrophic gastritis. Gastroenterology 2000;1 18:239-40.

30 Lundell L, Havu N, Miettinen P, et al. Changes of gastric mucosal architecture during long-term omeprazole therapy: results of a randomized clinical trial. Aliment Pharmacol Ther 2006;23:639-47.

31 Eissele R, Brunner $G$, Simon B, et al. Gastric mucosa during treatment with lansoprazole: Helicobacter pylori is a risk factor for argyrophil-cell hyperplasia. Gastroenterology 1997;112:707-17.

32 Geboes K, Dekker W, Mulder CJ, et al. Long-term lansoprazole treatment for gastro-oesophageal reflux disease: clinical efficacy and influence on gastric mucosa. Aliment Pharmacol Ther 2001;15:1819-26.

33 Marzio L, Biasco G, Cifani F, et al. Short- and long-term omeprazole for the treatment and prevention of duodenal ulcer, and effect on Helicobacter pylori. Am J Gastroenterol 1995;90:2172-6.

34 Stolte M, Meining A, Schmitz JM, et al. Changes in Helicobacter pyloriinduced gastritis in the antrum and corpus during 12 months of treatment with omeprazole and lansoprazole in patients with gastro-oesophageal reflux disease. Aliment Pharmacol Ther 1998;12:247-53.

35 Schenk BE, Kuipers EJ, Klinkenberg-Knol EC, et al. Atrophic gastritis during long-term omeprazole therapy affects serum vitamin B12 levels. Aliment Pharmacol Ther 1999;13:1343-6.

36 Sanduleanu S, Jonkers D, De Bruine A, et al. Double gastric infection with Helicobacter pylori and non-Helicobacter pylori bacteria during acidsuppressive therapy: increase of pro-inflammatory cytokines and development of atrophic gastritis. Aliment Pharmacol Ther 2001;15:1163-75.

37 Uemura N, Okamoto S, Yamamoto S, et al. Helicobacter pylori infection and the development of gastric cancer. N Engl J Med 2001;345:784-9.

38 Hansen S, Vollset SE, Ardill JES, et al. Hypergastrinemia is a strong predictor of distal gastric adenocarcinoma among Helicobacter pylori infected persons. Gut 1997;41:A41

39 Malfertheiner P, Megraud F, O'Morain C, et al. Current concepts in the management of Helicobacter pylori infection-The Maastricht III Consensus Report 2005. Submitted, 2006.

40 Moayyedi P, Wason C, Peacock R, et al. Changing patterns of Helicobacter pylori gastritis in long-standing acid suppression. Helicobacter 2000;5:206-14.

41 Kuipers EJ, Nelis GF, Klinkenberg-Knol EC, et al. Cure of Helicobacter pylori infection in patients with reflux oesophagitis treated with long-term omeprazole reverses gastritis without exacerbation of reflux disease; results of a randomised controlled trial. Gut 2004;53:12-20.

42 Panter SJ, O'Flanagan H, Bramble MG, et al. Empirical use of antisecretory drug therapy delays diagnosis of upper gastrointestinal adenocarcinoma but does not effect outcome. Aliment Pharmacol Ther 2004;19:981-8.

43 Bramble MG, Suvakovic Z, Hungin AP. Detection of upper gastrointestinal cancer in patients taking antisecretory therapy prior to gastroscopy. Gut 2000;46:464-7.

44 Lassen A, Hallas J, de Muckadell OB. The risk of missed gastroesophageal cancer diagnoses in users and nonusers of antisecretory medication. Gastroenterology 2005; 129:1179-86.

45 Wayman J, Hayes N, Raimes SA, et al. Prescription of proton pump inhibitors before endoscopy. A potential cause of missed diagnosis of early gastric cancers. Arch Fam Med 2000:385-8. 